\title{
Improvement of a Gaseous Position Sensitive Detector for use in Thermal Neutron Tomographic Systems
}

\author{
Marcelo J. Gonçalves, Ricardo T. Lopes, \\ Universidade Federal do Rio de Janeiro, COPPE \\ Centro de Tecnologia, Bloco 6, 21945-970, Rio de Janeiro,RJ, Brazil \\ Maria Ines Silvani, Gevaldo L. de Almeida, and Rosanne C. A. A. Furieri \\ Instituto de Engenharia Nuclear, CNEN \\ C.P. 68550, Ilha do Fundão, 21945-970, Rio de Janeiro, RJ, Brazil
}

Received on 9 September, 2003

\begin{abstract}
The utilization of a position sensitive detector in tomographic systems is an attractive possibility because it is capable of furnishing the position where the ionizing event occurs. This feature can reduce significantly the image acquiring time, since a sample translation is no longer required. In this work the performance of a gaseous position sensitive detector equipping a thermal neutron tomographic system has been improved by a stepwise increase of the filling-gas $\left({ }^{3} \mathrm{He}\right.$-enriched helium) pressure from 3 to 6 atm. Important quantitative detector parameters such as resolution, linearity and homogeneity have been measured for that pressure range, and compared with the tomographic images of test-samples. Several test-samples have been studied, all of them constituted by an aluminum cylinder containing inserts of different materials. Besides that, the modulation transfer function-MTF for the system has been experimentally obtained and compared with the expected theoretical curve. An improvement of both detector efficiency and resolution has been observed, as theoretically expected from an increase of the filling-gas pressure.
\end{abstract}

\section{Introduction}

The computer assisted tomography (CAT) is based on a quantitative mapping of the absorption coefficient of a single or multiple cross-sections of an object. The map is obtained by measuring the attenuation of a radiation beam passing through the object. Assigning colors to the obtained values, $2 \mathrm{D}$ or $3 \mathrm{D}$ images are obtained, depending whether a single or several cross-sections are inspected. A tomographic system is basically constituted by a set source-detector.

The system described in this work uses the Argonauta reactor at the Instituto de Engenharia Nuclear$C N E N$ as source of thermal neutrons, which yields a flux of $4.5 \times 10^{5} \mathrm{n} . \mathrm{cm}^{-2} . \mathrm{s}^{-1}$, while the radiation detection is carried out by a gaseous position sensitive detector operating at the proportional region. This detector has been filled up with ${ }^{3} \mathrm{He}$-enriched helium, for this gas exhibits several advantages in the detection of thermal neutrons, such as: low conversion efficiency for $\gamma$-rays [1], large cross-section and stopping power for charged particles even for low pressures.

The interaction of thermal neutrons with ${ }^{3} \mathrm{He}$ causes the nuclear reaction ${ }^{3} \mathrm{He}(\mathrm{n}, \mathrm{p})^{3} \mathrm{H}$. Due to the low neutron energy (about $0.025 \mathrm{eV}$ ) the reaction products are isotropically emitted into opposite directions, with proton and tritium carrying 573 and $191 \mathrm{keV}$ respectively.

The efficiency of a gaseous detector depends on the number of ions pairs produced by each incident neutron hitting the detector active volume. In the formerly mentioned reaction, the proton has a range of $7.9 \mathrm{~mm}$, while the tri- tium has approximately a half of this value, for a 10 atmpressure of ${ }^{3} \mathrm{He}$ [2]. The detector used in this work, filled up with $99 \%{ }^{3} \mathrm{He}$-enriched helium under $3 \mathrm{~atm}$-pressure, has a gap of $3 \mathrm{~mm}$ between its window and the and anode wire. Therefore, the charged particles produced in the gas would hardly loose all their energy inside the detector. The solution adopted in this work to overcome this handicap was to increase the filling-gas pressure, for this would produce a higher density of ions pairs, improving hence the detection efficiency.

Each one of the particles arising from the nuclear reaction generates an ionization track which centroid is displaced from the position where the reaction actually occurred due to their different ranges. This displacement, e as shown in Fig. 1, degrades the detector resolution. An increase of the filling-gas pressure, would cause a decrease in the range of those particles, reducing that displacement.

To evaluate the performance of the detection system, as well as that of the detector itself, some parameters such as resolution (RES), linearity and homogeneity [3] have been measured.

The detector resolution is defined by the full width at half maximum (FWHM) of the line spread function (LSF) which represents the detector response to an infinitely narrow slab-like radiation beam. Since in practice its not possible to obtain such a kind of beam, the detector response is assumed to follow a Gaussian function, and its resolution expressed as: 


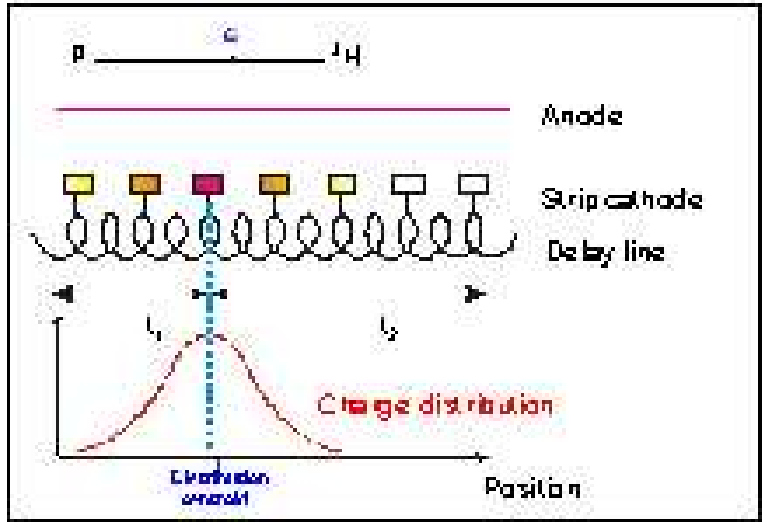

Figure 1. Resolution degradation caused by the displacement of the charge distribution centroid.

$$
R E S=\sqrt{F W H M^{2}-\Delta^{2}}
$$

where $\Delta$ is the width of the incident radiation beam.

The linearity expresses the deviation from a straight line of the curve relating the position where the incident radiation hits the detector causing the first ionizing events, and the position furnished by it.

The homogeneity evaluates the detector behavior when submitted to different counting statistics, by comparing the efficiency of its individual virtual cells.

Regarding the tomographic system, a measurable parameter used to evaluate quantitatively the spatial resolution is the modulation transfer function (MTF). This curve can be obtained from the discrete Fourier Transform of the line spread function (LSF) [4]. In other words, the MTF can be figured out as the result of a modulation imposed to an input signal by the tomographic system, due to its variable response to different spatial frequencies [5].

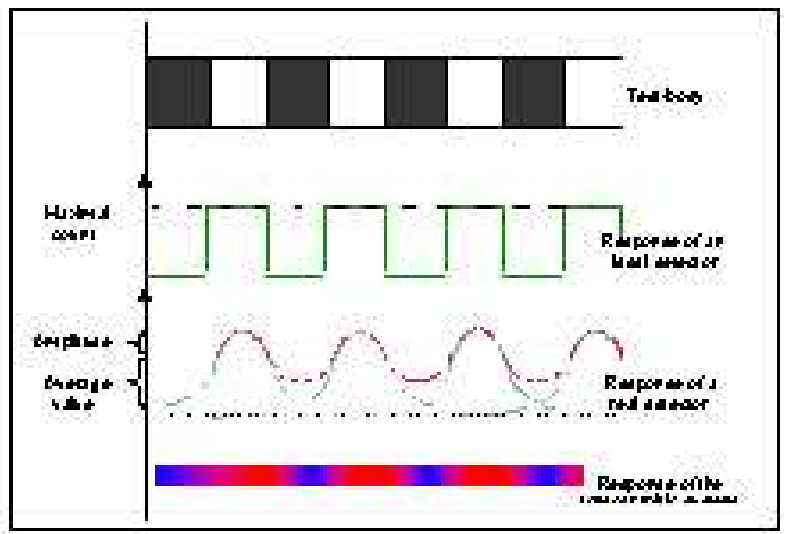

Figure 2. Impact of the spatial resolution on the final response of a tomographic system.

This modulation affects the response of the detector with a direct impact on the spatial resolution of a tomographic system. This is shown in Fig. 2, where an opaque test-body provided with three apertures is exposed to a radiation field. The expected response for a real detector placed behind the test-body is a convolution of Gaussian functions representing its LSF. One can observe a loss of contrast between the absorbing and non-absorbing regions. This loss depends on the spatial frequency of the test-body and on the capability of the system to deal with it [6].

The modulation is defined as the ratio of the amplitude to the average value. It is usually agreed that a modulation below $10 \%$ would not produce images of an acceptable quality [4].

\section{Experimental}

Within the frame of this work, addressing an improvement of the image quality and a reduction of its acquiring time, the gas pressure has been increased, to improve both resolution and efficiency. For this purpose, the original structure of the detector has been modified to withstand pressures in excess of $10 \mathrm{~atm}$. One of these modifications included the replacement of the detector front flange containing its window, by a single aluminum piece provided with a $1.7 \mathrm{~mm}$ thick window cast on it. This new flange should assure that no deformation would take place after a pressure increase.

The parameters characterizing the tomographic system, as well as its detector, have been obtained through a proper collimation of the neutron beam using gadolinium masks fitted to the detector window. To assess the impact of those parameters on the quality of the final images, some tomographic images of different test-bodies have been acquired.

\section{Results and Discussion}

After the changes in the detector filling-gas pressure, a fair improvement of its performance can be observed, as shown in Table I.

TABLE I. Detector performance under different filling-gas pressures, as experimentally measured.

\begin{tabular}{cccc}
\hline $\begin{array}{c}\text { Pressure } \\
{ }^{3} \mathrm{He}\end{array}$ & $\begin{array}{c}\text { Resolution } \\
(\mathrm{mm})\end{array}$ & $\begin{array}{c}\text { Linearity } \\
(\%)\end{array}$ & $\begin{array}{c}\text { Homogeneity } \\
(\%)\end{array}$ \\
\hline $3 \mathrm{~atm}$ & 1.70 & 0.9 & 9 \\
$4 \mathrm{~atm}$ & 1.32 & 0.7 & 6 \\
$6 \mathrm{~atm}$ & 1.1 & 0.6 & 6 \\
\hline
\end{tabular}

Regarding the tomographic system, the appropriate characterization is given by the MTF curve which has been obtained by a Fourier transform of a Gaussian function taken as a LSF of the detector. The results for the system using detectors under different pressures are shown in Table II.

TABLE II. Results inferred from a theoretical MTF obtained after a Fourier transform of the detector LSF.

\begin{tabular}{cccc}
\hline $\begin{array}{c}\text { Gas } \\
\text { Pressure }\end{array}$ & $3 \mathrm{~atm}$ & $4 \mathrm{~atm}$ & $6 \mathrm{~atm}$ \\
\hline $10 \%$ cutoff & $3.84 \mathrm{~mm}$ & $3.58 \mathrm{~mm}$ & $3.10 \mathrm{~mm}$ \\
\hline
\end{tabular}

The above values represent the minimal gap between two features in the object being analyzed, capable of being distinguished and recognized as that, on an image of reasonable quality. 


\section{Tomographic images}

The tomographic images taken as examples to evaluate the impact of the gas pressure on the detector resolution are shown in Fig. 3. They refer to a $22 \mathrm{~mm}$-diameter teflon cylinder containing seven orifices filled up with $\mathrm{Gd}_{2} \mathrm{O}_{3}$. The orifice diameters are within the range 1-4 $\mathrm{mm}$.
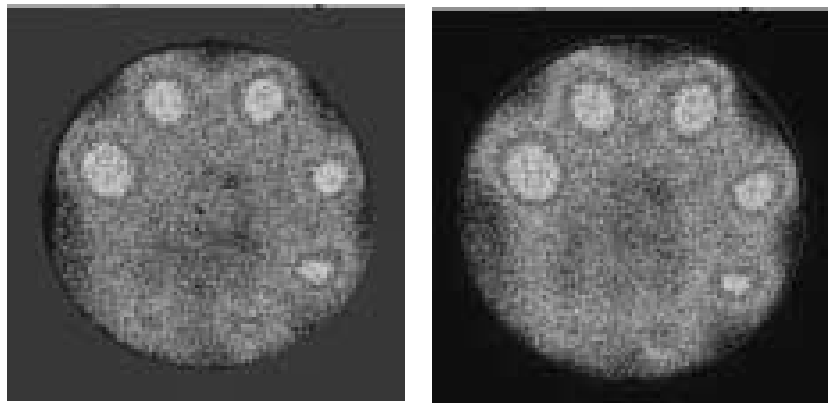

Figure 3. Tomographic images taken with detectors under 4 atm (left) and $6 \mathrm{~atm}$ (right) gas pressures.
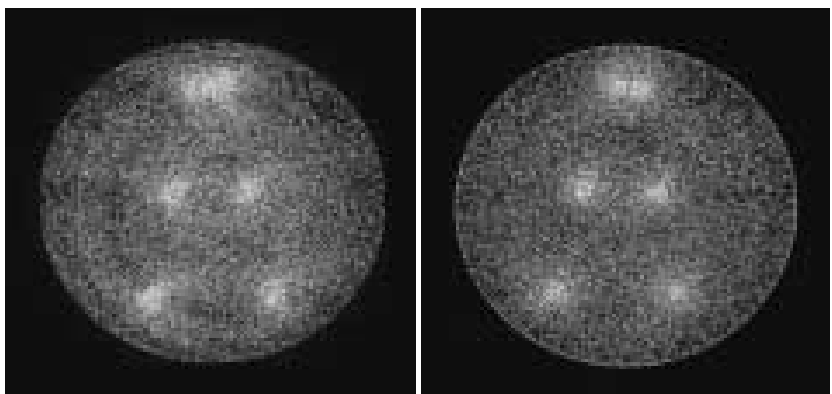

Figure 4. Tomographic images taken with systems equipped with ${ }^{3} \mathrm{He}$-filled PSD under 4 atm (left) and 6 atm (right).

Figure 4 shows tomographic images of a $22 \mathrm{~mm}$ diameter aluminum cylinder provided with 3 pairs of orifices containing $1 \mathrm{~mm}$-diameter cadmium wires.

The gaps between the orifices for each pair are 2,6 and $10 \mathrm{~mm}$. By observing the images taken with the 4 and 6 atm systems, under a statistics of 4,000 counts/channel, one can recognize a better resolution and contrast of that acquired with a 6 atm detector.

As far as efficiency is concerned, both systems exhibit an improvement with the gas pressure, as theoretically expected. Table III shows the spent time required to acquire the above mentioned images, for 90 projections and a 4,000 counts/channel statistics.

TABLE 3. Spent time demanded to achieve 4000 counts/channel using the studied systems.

\begin{tabular}{cccc}
\hline Pressure & $3 \mathrm{~atm}$ & $4 \mathrm{~atm}$ & $6 \mathrm{~atm}$ \\
\hline Time (min) & 90 & 70 & 60 \\
\hline
\end{tabular}

\section{Applications}

Neutron and x-rays tomography are intrinsically complementary techniques. Therefore, one can hardly replace the other, for the basic parameter which commands their potential and capabilities - the attenuation coefficient of the involved materials - is dictated by nature. One example where the use of x-rays tomography would fail, or at least would not present good results, while the neutron tomography would work very well, is presented in Fig. 5. The image refers to an aluminum cylinder containing orifices filled up with plastic or hydrogen-bearing materials, which thanks to their high cross-section for thermal neutrons are easily detected, and thus, clearly visualized in the tomographic image.

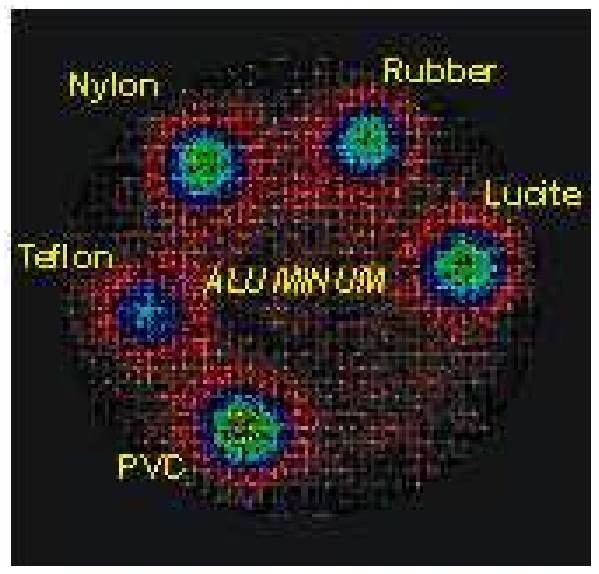

Figure 5. Tomographic image of an aluminum cylinder containing rods of plastic materials.

\section{Conclusions}

The utilization of thermal neutrons in tomographic systems produces satisfactory results, making feasible the using of this approach as a complementary technique to the nondestructive assay utilizing $\mathrm{x}$-rays or gamma radiation. Furthermore, the incorporation of a position sensitive detector to the tomographic system is an attractive and advantageous option because a sample or source-detector translation would no longer be necessary, reducing therefore the image acquisition time. A consequent disadvantage associated with the PSD, is a limitation on the maximal size of the object being analyzed, which cannot obviously exceed the detector length. In this work, this limit was $8 \mathrm{~cm}$.

The use of filling-gas under higher pressures, results in a better detector resolution, which improves the quality of the image furnished by the tomographic system, making it capable to detect otherwise non-detectable small features. Another achievement is the reduction on the image acquiring time, a direct consequence of the better efficiency of the detectors submitted to higher pressures. This aspect is very important, for it reduces costs and the inevitable radiological burden imposed to the staff involved in the research itself, as well as in the reactor operation. 


\section{References}

[1] M. I. Silvani, Computer Tomography with Thermal Neutrons and Position Sensitive Detector DSc. Thesis, COPPE/UFRJ, Rio de Janeiro, RJ, Brazil. (2001) In Portuguese.

[2] J. Alberi, et al. Physics Research B 127, 507 (1975).

[3] A. F. Barbosa, CBPF-NF068/94, Rio de Janeiro, Brazil (1994).

[4] ASTM E 1441-95 and 1570-95a, Non-Destructive Testing, Radiation Methods, Computed Tomography. Guide for Imaging and Practice for Examination, ISO/TC 135/SC 5, N 118, USA (1996).

[5] G. L. de Almeida, M. I. Silvani, and R. T. Lopes, August 2002, A Hybrid Technique to Evaluate the Line Spread Function. VI ENAN, E10-142, Rio de Janeiro, Brazil, (2002).

[6] M. J. Gonçalves, Optimization of a gaseous position sensitive detector for use in a thermal neutron tomographic system. M.Sc. Thesis, COPPE / UFRJ, Rio de Janeiro, RJ, Brazil (2003). In Portuguese. 